\title{
Interrogating the impact of KIR ligand mismatch in engraftment following HLA-disparate stem cell transplantation
}

\author{
Lucy Li $\mathbb{C}^{1} \cdot$ Merle Kolk ${ }^{2} \cdot$ Marcelo Fernandez-Vina ${ }^{3} \cdot$ Marcos de Lima $\mathbb{C}^{2} \cdot$ Folashade Otegbeye $\mathbb{C}^{4}$
}

Received: 17 March 2020 / Revised: 15 May 2020 / Accepted: 18 May 2020 / Published online: 27 May 2020

(c) The Author(s), under exclusive licence to Springer Nature Limited 2020

\begin{abstract}
The effects of donor-derived natural killer (NK) cell alloreactivity on disease relapse and transplant-related mortality following allogeneic stem cell transplantation have been described while the impact of recipient-derived NK cell alloreactivity on donor engraftment is not well known. Epitopes of HLA Class I molecules act as ligands for NK cell killer immunoglobulin-like receptors (KIR) regulating their cytotoxicity. As such, NK cell alloreactivity is predictable from KIR ligand mismatches between donors and recipients. We analyzed the impact of KIR ligand mismatch (KIR-L-MM) on donor engraftment in 70 cord blood transplants (CBT) and 26 haploidentical transplants (HaploSCT). In CBT, host-versus-graftdirected KIR-L-MM predicted primary graft failure; an effect not mitigated by use of ATG. This trend was most significant with HLA-C KIR-L-MM. In addition, graft-versus-host-directed KIR-L-MM predicted the dominant cord blood unit in double CBT. In the limited HaploSCT cohort, host-versus-graft-directed KIR-L-MM did not predict graft failure. Time to neutrophil engraftment was unaffected by KIR-L-MM in either CBT or HaploSCT. The direction of KIR-L mismatch may be a parameter to consider when selecting CBT units to ensure successful engraftment. The role of KIR-L-MM in CBT and HaploSCT engraftment merits further exploration in a large transplant database.
\end{abstract}

\section{Introduction}

There are specific epitopes of the HLA Class I molecules HLA-B and HLA-C presented as ligands to inhibitory killer immunoglobulin-like receptors (KIRs), which regulate natural killer (NK) cell cytotoxicity. Because NK cell licensing is thought to select for circulating NK cells with inhibitory KIRs for self KIR ligands (KIR-L), the incompatibility in donor versus recipient KIR-L has been used as a surrogate predictor of NK cell alloreactivity in HLA-disparate transplants [1-3].

Folashade Otegbeye

folashade.otegbeye@uhhospitals.org

1 Case Western Reserve University School of Medicine, Cleveland, OH, USA

2 Stem Cell Transplant Program, University Hospitals Seidman Cancer Center, Cleveland, OH, USA

3 Department of Pathology, Stanford University School of Medicine, Palo Alto, CA, USA

4 Stem Cell Transplant Program, University Hospitals Seidman Cancer Center, 11100 Euclid Avenue, Cleveland, OH 44106, USA
There is plausible, though varied, clinical evidence suggesting the benefit of donor-derived NK cell alloreactivity in outcomes after allogeneic hematopoietic stem cell transplantation (HSCT) [1-7]. Recipient-derived NK cell alloreactivity on the other hand could potentially impact engraftment, resulting in primary graft failure (PGF) or delayed engraftment. In an EBMT/Center for International Blood and Marrow Transplant Research (CIBMTR) study of single-cord blood transplants (CBT) for acute myeloid leukemia, host-versus-graft (HvG)directed KIR ligand mismatch (KIR-L-MM) was associated with increased nonrelapse and overall mortality [4]. The incidence of graft failure or its associations was not reported.

The potential directions of NK cell alloreactivity in HLA-disparate transplants, such as CBT and haploidentical transplants (HaploSCT), are graft-versus-host $(\mathrm{GvH})$ and $\mathrm{HvG}$ in both and graft-versus-graft $(\mathrm{GvG})$ in CBT. We hypothesized that HvG-directed NK cell alloreactivity can drive engraftment outcomes in CBT and HaploSCT and that GvG interactions would predict the dominant graft in double CBT (dCBT). We explored these hypotheses in a small number of CBT and HaploSCT performed at a single center. 


\section{Materials and methods}

The Institutional Review Board of University Hospitals Cleveland Medical Center approved this retrospective study protocol. We analyzed the Stem Cell Transplant database of all CBT and HaploSCT performed at University Hospitals Seidman Cancer Center between December 2010 and April 2018. Data extracted from electronic medical record review included demographics, indication for transplant, disease status at time of transplant, conditioning regimen, graft-versus-host disease (GVHD) prophylaxis including the use of antithymocyte globulin (ATG) and post transplantation outcomes. Analysis was limited to CBT and HaploSCT for which allelelevel HLA typing were available for donors and recipients to enable correct KIR-L identification. Determination of donor/recipient KIR-L and the resulting directions of NK cell alloreactivity were computed using the KIR-L calculator available online (https://www.ebi.ac.uk/ipd/kir/ ligand.html). Patients that died within the first 30 days of CBT or HaploSCT were also excluded from analysis. We determined the relative risk of PGF as well as summary statistics of time to neutrophil engraftment and achieving a stable lymphocyte count of $500 / \mu 1$ or more in those that engrafted. Comparisons of relative risk between groups were considered statistically significant at $p$ value $<0.05$.

\section{Results}

We identified 67 patients that received CBT and 26 HaploSCT for which donor and recipient KIR-L could be adequately documented. Sixty-four CBT patients received only one transplant during the time period reviewed, while three patients required a second CBT due to PGF of the first, resulting in a total of 70 CBTs for the cohort. Demographics, disease, characteristics and treatment regimens for both CBT and HaploSCT are outlined in Table 1. The median (range) total nucleated cell (TNC) dose of cord blood units was $2.39(1.03-40) \times 10^{7} / \mathrm{kg}^{-1}$ body weight of the recipient. In patients older than 16 years, TNC doses were between 1.5 and $4 \times 10^{7} / \mathrm{kg}$ TNC. Cord blood units were matched at HLA-A, -B, -C, and DRB1 at a median of $6 / 8$ with matches occurring at $3 / 8(2 \%), 4 / 8(8.5 \%), 5 / 8$ (29\%), 6/8 (37\%), $7 / 8(15 \%)$, and $8 / 8(8.5 \%)$.

\section{HvG-directed KIR-L-MM predicts primary graft failure (PGF), while GvH-directed KIR-L-MM may indicate the dominant cord unit in CBT}

There were KIR-L mismatches (MM) in the $\mathrm{HvG}$ and GvH direction in 32 and 31 recipient-cord blood unit pairs,
Table 1 Baseline demographics, disease, and treatment variables.

\begin{tabular}{|c|c|c|}
\hline & $\mathrm{CBT}(\%)$ & Haplo $(\%)$ \\
\hline \multicolumn{3}{|l|}{ Gender } \\
\hline Female & $31(46)$ & $16(62)$ \\
\hline Male & $36(54)$ & $10(38)$ \\
\hline \multicolumn{3}{|l|}{ Age at transplant (years) } \\
\hline$<1-29$ & $18(27)$ & $10(38)$ \\
\hline $30-45$ & $9(13)$ & $4(15)$ \\
\hline $46-60$ & $26(39)$ & $9(35)$ \\
\hline $61-71$ & $14(21)$ & $3(12)$ \\
\hline \multicolumn{3}{|l|}{ Transplant diagnosis } \\
\hline AML/MDS & $39(58)$ & $12(46)$ \\
\hline ALL & $7(10)$ & $4(15)$ \\
\hline Lymphoma/CLL & $9(13)$ & $1(4)$ \\
\hline Nonmalignant & $6(9)$ & $3(12)$ \\
\hline CML & $3(5)$ & $4(15)$ \\
\hline Myelofibrosis/JMML & $3(5)$ & $2(8)$ \\
\hline \multicolumn{3}{|c|}{ Disease status at transplant (malignancy only) } \\
\hline Persistent or MRD & $16(26)$ & $4(19)$ \\
\hline No disease & $45(74)$ & $17(81)$ \\
\hline \multicolumn{3}{|l|}{ Conditioning regimen } \\
\hline Fludarabine/Melphalan & $21(30)$ & $1(4)$ \\
\hline Fludarabine/Cyclophosphamide/TBI & $34(48)$ & $17(65)$ \\
\hline Busulfan/Cyclophosphamide & $11(16)$ & $0(0)$ \\
\hline Other & $4(6)$ & $8(31)$ \\
\hline \multicolumn{3}{|l|}{ ATG/Alemtuzumab } \\
\hline ATG & $49(70)$ & $3(12)$ \\
\hline Alemtuzumab & $0(0)$ & $4(15)$ \\
\hline Neither & $21(30)$ & $19(73)$ \\
\hline \multicolumn{3}{|l|}{ GVHD prophylaxis } \\
\hline Tacrolimus + MMF & $33(47)$ & $11(42)$ \\
\hline Cyclosporine $+\mathrm{MMF}$ & $37(53)$ & $0(0)$ \\
\hline $\mathrm{PTCY}+$ tacrolimus $+\mathrm{MMF}$ & $0(0)$ & $12(46)$ \\
\hline Sirolimus + MMF & $0(0)$ & $1(4)$ \\
\hline Tacrolimus + other & $0(0)$ & $2(8)$ \\
\hline
\end{tabular}

$A M L$ acute myeloid leukemia, $M D S$ myelodysplastic syndrome, $A L L$ acute lymphocytic leukemia, CLL chronic lymphocytic leukemia, $C M L$ chronic myeloid leukemia, JMML juvenile myelomonocytic leukemia, MRD minimal residual disease, TBI total body irradiation, $A T G$ antithymocyte globulin, $M M F$ mycophenolate mofetil, PTCY posttransplant cyclophosphamide.

respectively. There were no KIR-L MM in $26 \mathrm{CBT}$, while 7 had bidirectional KIR-L MM.

Overall, 16 CBT failed to engraft (23\%). There was an increased risk of PGF in recipients of HvG-directed KIR-L MM transplants (relative risk (R.R) 2.15; $p=0.063$ ) with this trend most significant when the mismatch was in HLA-C ligand (R.R 2.46; $p=0.027$ ); (Table 2). Of eight dCBTs in which both cord units were KIR-L mismatched in the HvG direction, three failed to engraft (37.5\% PGF). Of eight 
Table 2 Relative risk of primary graft failure in CBT cohort.

\begin{tabular}{lll}
\hline KIR ligand mismatch (KIR-L-MM) category & Relative risk (95\% C.I) & $p$ value \\
\hline KIR-L-MM in any direction $(n=44)$ & $1.62(0.64-4.07)$ & 0.305 \\
KIR-L-MM in HvG direction $(n=31)$ & $2.15(0.959-4.828)$ & 0.063 \\
KIR-L-MM in HvG direction with C Ligand $(n=27)$ & $2.46(1.11-5.49)$ & 0.027 \\
KIR-L-MM in HvG direction with B Ligand $(n=6)$ & $1.68(0.67-4.18)$ & 0.266 \\
KIR-L-MM in GvH direction $(n=23)$ & $0.64(0.23-1.76)$ & 0.384 \\
\hline
\end{tabular}

Table 3 Time (in days) to stable neutrophil recovery in CBT patients without graft failure.

\begin{tabular}{|c|c|c|c|c|c|}
\hline & $\begin{array}{l}\text { No KIR- } \\
\text { L MM }\end{array}$ & $\begin{array}{l}\text { KIR-L MM } \\
(\mathrm{HvG} \text { and } \mathrm{GvH})\end{array}$ & $\begin{array}{l}\text { HvG KIR- } \\
\text { L MM }\end{array}$ & $\begin{array}{l}\text { GvH KIR- } \\
\text { L MM }\end{array}$ & \\
\hline \multicolumn{6}{|c|}{ ANC500/ $\mu \mathrm{L}$} \\
\hline Mean & 22.65 & 26.07 & 25.54 & 25.95 & $p=0.14$ \\
\hline Median & 21 & 26 & 25 & 25 & \\
\hline Range & $13-42$ & $14-64$ & $14-46$ & $15-64$ & \\
\hline \multicolumn{6}{|c|}{ 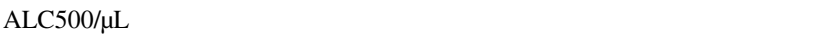 } \\
\hline Mean & 47.44 & 53.62 & 48.58 & 55.06 & $p=0.61$ \\
\hline Median & 38.5 & 46 & 44 & 48 & \\
\hline Range & $21-94$ & $25-141$ & $28-104$ & $25-141$ & \\
\hline
\end{tabular}

dCBT where one KIR-L mismatched in the HvG direction, the nonmismatched unit fully engrafted in seven (87.5\%) cases. In one case, the KIR-L-matched unit shared donor chimerism with the KIR-L MM unit at $85 \%$ and $15 \%$, respectively, till 10 months post transplant following which the KIR-L-matched unit fully engrafted. Four of six dCBT (67\%) engrafted the GvH-directed KIR-L-MM cord unit.

In the entire CBT cohort when primary engraftment occurred, the time to neutrophil engraftment and stable lymphocyte recovery was not significantly impacted by KIR-L-MM (Table 3).

\section{In double CBT, the cord unit with KIR-L-MM directed alloreactivity against the recipient or the alternate graft was more frequently the dominant unit}

In six dCBTs where there was KIR-L-MM from one 1 cord unit in the $\mathrm{GvH}$ and the $\mathrm{GvG}$ (graft versus other graft) direction, $4(67 \%)$ of such units became the dominant graft.

\section{KIR-L-MM did not predict primary graft failure in $\mathbf{2 6}$ patients who received HaploSCT in the same evaluation period}

There were $14 \mathrm{GvH}$-directed KIR-L-MM pairs, nine HvGdirected KIR-L-MM donor-recipient pairs (two mismatched in both $\mathrm{HvG}$ and $\mathrm{GvH}$ direction) and three KIR-ligandmatched pairs (Table 1). Five transplants in this entire cohort failed to engraft (19\%) with all PGF being KIR-L-MM in the GvH direction. The incidence of PGF in
GvH KIR-L-MM in this cohort was therefore $35.7 \%$ with R.R 9.53; $p=0.114$. All nine HvG KIR-L-MM pairs and the two transplant pairs KIR-L mismatched in both HvG and $\mathrm{GvH}$ directions engrafted. Time to neutrophil engraftment was not different for GvH or HvG KIR-L-MM (data not shown).

\section{The use of T-lymphocyte depletion with antithymocyte globulin for CBT does not ameliorate risk of PGF in HvG-directed KIR-L-MM}

Given the HLA-mismatched nature of CBT, we investigated if alloreactive host $\mathrm{T}$ cells would be more important than alloreactive host NK cells in graft rejection. In this case, PGF would be ameliorated in CBT patients who have HvGdirected KIR-L MM and are conditioned with ATG. However, in our cohort, those patients actually had an even higher rate of PGF: $44.4 \%$ (8/18) compared with $3.7 \%$ $(1 / 27)$ in those without HvG KIR-L-MM who also received ATG.

\section{Discussion}

Alternative donor transplantation using umbilical cord blood (CBT) or HLA-haploidentical donors (HaploSCT) has significantly increased access to allogeneic stem cell transplantation for a variety of diseases. Where multiple such donors are available for a patient, it would be important to guide donor selection using readily available criteria, such as routinely done HLA typing, that can predict preferred outcomes. In this small, single center experience of CBT and HaploSCT, we explored the impact of KIR-L-MM on engraftment to see if the predicted direction of NK cell alloreactivity merits further investigation as a donor selection criterion.

When predicting NK cell alloreactivity based on KIR-L$\mathrm{MM}$, this study suggests an increased likelihood of graft failure in CBT when HvG KIR-L-MM exists. There is also a trend in favor of engrafting the GvH/GvG KIR-L-MM unit in dCBT. While ATG during conditioning therapy would deplete alloreactive host $\mathrm{T}$ cells in HLA-disparate HSCT, its use in this CBT did not mitigate the effect of HvG NK cell alloreactivity on PGF. TNC dose of the cord units transplanted did not appear to be a significant factor in our CBT with PGF. The median TNC dose administered in 
the nineteen PGF was $2.28 \times 10^{7} / \mathrm{kg}$ (median $2.38 \times 10^{7} / \mathrm{kg}$ in the entire cohort). Our small cohort size limits in-depth multivariate analysis accounting for cell dose, categories of HLA mismatch, use of ATG/Alemtuzumab, intensity of conditioning regimen, and other potential confounders.

A similar graft failure effect of HvG KIR-L-MM was not seen in our HaploSCT cohort; however, interpreting this finding is limited by the even smaller cohort size $(N=26)$. It is unclear if our reverse finding of PGF only in the GvHdirected KIR-L-MM transplant pairs represents a true finding or simply incidental due to the sampling limitation. At the time of this analysis, only 11 patients $(42 \%)$ received posttransplant cyclophosphamide (PTCY) as GVHD prophylaxis for HaploSCT. As the prevalent clinical practice of GVHD prophylaxis in HaploSCT currently includes PTCY, the association between KIR-L-MM and PGF in this setting merits exploration in a larger PTCY database.

We plan to analyze, in more detail, these preliminary findings associating KIR-L-MM with engraftment in the large, multi-institutional transplant database of the CIBMTR. This study proposal is currently in development with the Immunobiology Working Committee of the CIBMTR. In this setting, we would be sufficiently powered to account for multiple confounding variables such as intensity of conditioning, number/pattern of HLA-mismatches, cell dose and use of lymphocyte-depleting strategies before incorporating KIR-L-MM direction in our donor selection process for transplantation from cord blood or haploidentical donors.

Acknowledgements Departmental funding through the Hematology and Oncology Division of University Hospitals Cleveland Medical Center supports the authors' work.

\section{Compliance with ethical standards}

Conflict of interest The authors declare that they have no conflict of interest.

Publisher's note Springer Nature remains neutral with regard to jurisdictional claims in published maps and institutional affiliations.

\section{References}

1. Ruggeri L, Capanni M, Casucci M, Volpi I, Tosti A, Perruccio K, et al. Role of natural killer cell alloreactivity in HLA-mismatched hematopoietic stem cell transplantation. Blood. 1999;94:333-9.

2. Ruggeri L, Mancusi A, Capanni M, Urbani E, Carotti A, Aloisi T, et al. Donor natural killer cell allorecognition of missing self in haploidentical hematopoietic transplantation for acute myeloid leukemia: challenging its predictive value. Blood. 2007;110:433-40.

3. Ruggeri L, Capanni M, Urbani E, Perruccio K, Shlomchik WD, Tosti A, et al. Effectiveness of donor natural killer cell alloreactivity in mismatched hematopoietic transplants. Science. 2002;295:2097-100.

4. Rocha V, Ruggeri A, Spellman S, Wang T, Sobecks R, Locatelli F, et al. Killer cell immunoglobulin-like receptor-ligand matching and outcomes after unrelated cord blood transplantation in acute myeloid leukemia. Biol Blood Marrow Transplant. 2016;22: 1284-9.

5. Mancusi A, Ruggeri L, Urbani E, Pierini A, Massei MS, Carotti A, et al. Haploidentical hematopoietic transplantation from KIR ligand-mismatched donors with activating KIRs reduces nonrelapse mortality. Blood. 2015;125:3173-82.

6. Willemze R, Rodrigues CA, Labopin M, Sanz G, Michel G, Socie $\mathrm{G}$, et al. KIR-ligand incompatibility in the graft-versus-host direction improves outcomes after umbilical cord blood transplantation for acute leukemia. Leukemia. 2009;23:492-500.

7. Giebel S, Locatelli F, Lamparelli T, Velardi A, Davies S, Frumento G, et al. Survival advantage with KIR ligand incompatibility in hematopoietic stem cell transplantation from unrelated donors. Blood. 2003;102:814-9. 